\title{
ADAPTATION FOR FREQUENCY FOCUSING AND INCREASED SENSITIVITY IN BIOMIMETIC FLOW SENSORS USING ELECTROSTATIC SPRING SOFTENING
}

\author{
J. Floris, N. Izadi, R.K. Jaganatharaja, R.J. Wiegerink, T.S.J. Lammerink and G.J.M. Krijnen ${ }^{1}$ \\ $\mathrm{Mesa}^{+}$Research Institute, University of Twente, Enschede, THE NETHERLANDS \\ ( ${ }^{1}$ Tel: +31 53489 4422; g.j.m.krijnen@ewi.utwente.nl)
}

\begin{abstract}
This paper presents the results of active adaptation of sensor sensitivity. By applying a DC-bias voltage to the sensing electrodes of a cricket inspired artificial hair sensor the effective spring stiffness can be adapted resulting in a reduced resonance frequency and increased sensitivity. An array of flow sensors was actuated using electrical and acoustical signals at different values of the DC-bias voltage. Characterization was done using a scanning laser vibrometer. Both resonance frequency versus applied DC-bias voltage and deflection-amplitude versus DC-bias voltage behave well in accordance to theory and show that adaptation by DC-biasing can be used for frequency focusing and increasing sensitivity.
\end{abstract}

Keywords: biomimetics, flow-sensors, adaptation, mechano-sensory hairs

\section{INTRODUCTION}

Crickets have filiform flow sensitive hairs in various lengths, diameters and directions on their abdominal appendages called cerci [1], see Fig.1. Each hair is attached to a sensory neuron at its base center, which is mechanically induced by stress due to hair deflection. These mechanoreceptor hairs have proven to be extremely flow sensitive [2], and enable crickets to detect complex flow patterns produced by, for instance, predators [3].

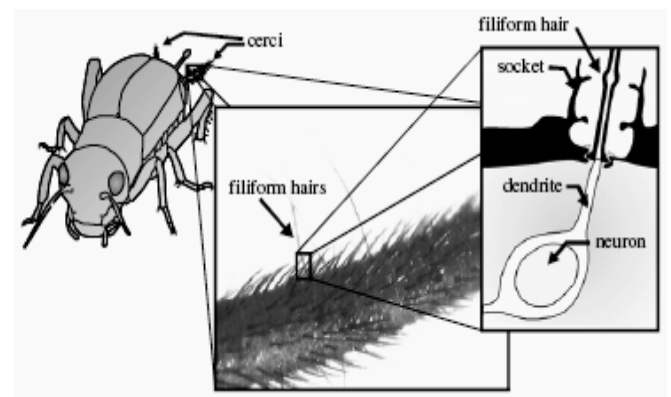

Fig. 1 Filiform flow sensitive hairs on the cerci of a cricket [1]

This research focuses on mimicking the filiform cricket hairs and their sensing abilities. Previously we have presented an array of SU-8 artificial sensory hairs on top of micromachined read-out structures [4]. To increase the sensitivity of these sensors with respect to previous work, the shape of substrate has been changed to a cercus-like structure to minimize the boundary layer [5] and the length of the hairs has been increased to about $1 \mathrm{~mm}$ to better escape the boundary layer effects, see Fig. 2.

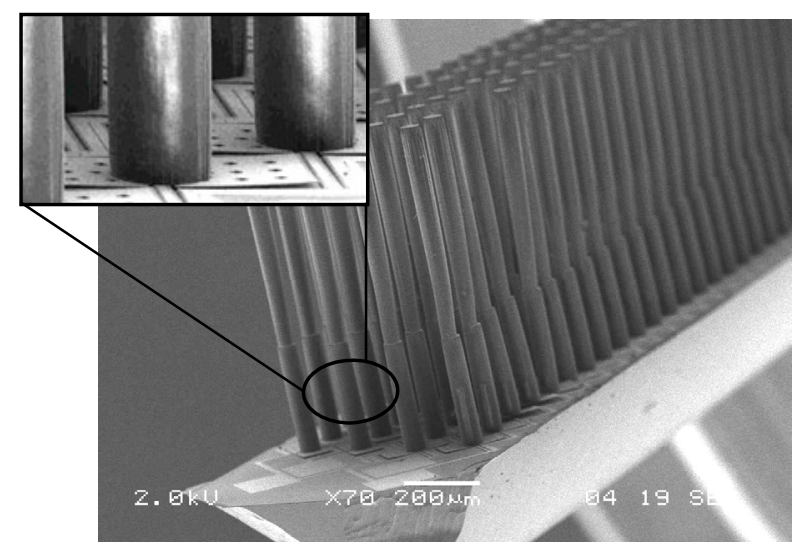

Fig. 2 SEM picture of an artificial cercus with $1 \mathrm{~mm}$ long sensory hairs. Inset: close up of the base showing slight curvature.

In this paper we focus on a third way to increase sensitivity: adaptation of the effective spring stiffness by DC-biasing the sensor structures, allowing for increased effective compliance of the sensor and tuning of the sensors resonance frequencies.

\section{OPERATING PRINCIPLE}

The hair-sensors in this work are fabricated by a combination of sacrificial poly-silicon technology 
with low-stress silicon-rich-nitride (SiRN) [6], for fabrication of suspended membranes, and SU-8 polymer processing for hair fabrication [4]. Using two $500 \mu \mathrm{m}$ thick SU-8 spin-exposure cycles, hairs with diameters of about $50 \mu \mathrm{m}$ and a length of approximately $1 \mathrm{~mm}$ are fabricated. For a detailed description of the fabrication process see [4]. A schematic of a single sensor is given in Fig. 3.

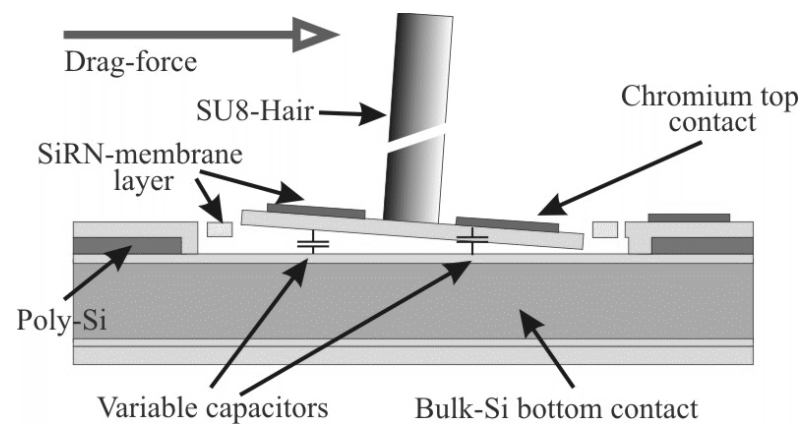

Fig. 3 Schematic of an artificial hair sensor

Flow induced drag torque on the hair forces tilting of the silicon nitride membrane. This results in a differential change of the two capacitances formed by the electrodes on top of the membrane and the substrate. For small angles of rotation these changes are proportional to the applied flow [4]. Read-out of drag-induced membrane-tilting can be done by capacitive measurements using charge amplifiers and synchronous detection. Using the sensor electrodes for actuation rather than for sensing, the parameters of the device can be dynamically changed and therefore deflection behavior can be adapted.

\section{ADAPTATION}

Transduction theory shows that the effective spring stiffness can be modulated by an applied bias voltage according to:

$$
S_{e f f}=S_{0}-\frac{U_{\text {bias }}^{2}}{2} \frac{\partial^{2} C}{\partial \alpha^{2}}=S_{0}-U_{\text {bias }}^{2} \kappa
$$

Where $S_{\text {eff }}$ is the effective spring constant, $\alpha$ is the membrane angular rotation, $S_{0}$ is the physical rotational spring stiffness of the torsional beams, $U_{\text {bias }}$ is the applied bias voltage, $C$ is the capacitance of the device and $\kappa$ is the second derivative of the capacitance with respect to the angle of rotation divided by two. Note that $U_{\text {bias }}{ }^{2}$ is always positive and therefore an applied bias voltage will always give a reduction in the spring stiffness. At frequencies sufficiently lower than the resonance frequency the effective spring stiffness variations will allow for adaptive sensitivity variations according to:

$$
\alpha=\frac{T\left(\omega, V_{0}\right)}{S_{\text {eff }}}=\frac{\alpha_{0}}{1-\frac{\kappa}{S_{0}} U_{\text {bias }}^{2}},
$$

where $T$ is the total drag torque on the hair (depending on frequency $\omega$ and far-field velocity amplitude $V_{0}$ ) and $\alpha_{0}$ is the angle of rotation for given $T$ without applied DC-bias. By the relation between resonance frequency and spring stiffness we expect a dependency of the resonance frequency, $\omega_{0}$, on the DC-bias voltage according to:

$$
\omega_{0}\left(U_{\text {bias }}\right)=\sqrt{\frac{S\left(U_{\text {bias }}\right)}{I}}=\omega_{0}(0) \sqrt{1-\frac{\kappa}{S_{0}} U_{\text {bias }}{ }^{2}}
$$

Here $\kappa / S_{0}$ is a constant depending on the geometry of the structure. From Eqs. (2) and (3) it follows that the deflection per unit drag torque of the sensors and the resonance frequency can be adaptively changed to accommodate optimal signal reception. Obviously, judging from Eqs. (2) and (3) it can be seen that the biasing effects are dependent on the ratio $\kappa / S_{0}$. Moreover, Eq (2) predicts that instability $\left(S_{\text {eff }}=0\right)$ will occur at a bias voltage of:

$$
S_{\text {eff }}=0 \quad \Rightarrow \quad U_{\text {bias }}= \pm \sqrt{\frac{S_{0}}{\kappa}}
$$

In order to estimate the sensitivity to DC-biasing we calculated the coefficient $\kappa$. In General this coefficient cannot easily be calculated analytically for any non-zero $\alpha$. However, in the case of the hair sensors studied here, with small angles of rotation encountered in practice, $\kappa$ is calculated as in Eq. (5) where $g$ is the spacing between the capacitance electrodes and $A$ the surface area of the electrodes.

$$
\kappa=\frac{\partial^{2}}{\partial \alpha^{2}}\left(\frac{1}{2} \int_{A} \frac{\varepsilon_{0} d A}{g(x, y, \alpha)}\right) \approx \lim _{\alpha \rightarrow 0} \frac{1}{2} \int_{A} \frac{\partial^{2}}{\partial \alpha^{2}}\left(\frac{\varepsilon_{0}}{g(x, y, \alpha)}\right) d A
$$


Using Eq. (5) we find for a stress-induced curved circular membrane, with radius $R$ and maximum deflection $a$ at the rim:

$$
\kappa=\frac{\pi \varepsilon_{0}}{8 a} R^{4}\left\{\frac{1}{t_{\text {eff }}^{2}}-\frac{1}{\left(t_{\text {eff }}+a\right)^{2}}\right\}
$$

Where $t_{\text {eff }}=d+\left(t_{S i R N}\right) / \varepsilon_{r}, \mathrm{~d}$ is the airgap and $t_{S i R N}$ is the total SiRN thickness in the capacitors.

\section{CHARACTERIZATION}

Two types of measurements were conducted on circular shaped sensors. Electrical actuation was used for determination of resonance frequencies at varying DC-biases and acoustic actuation was used for determination of air flow sensitivity versus DCbias. The specific dimensions of the sensor are given in table (1).

\section{Table 1. Sensor dimensions}

\begin{tabular}{|c|r|}
\hline Membrane (SiRN) & \\
\hline Diameter & $210 \mu \mathrm{m}$ \\
\hline Thickness & $1 \mu \mathrm{m}$ \\
\hline Gap spacing & $1 \mu \mathrm{m}$ \\
\hline Curvature $a_{\mathrm{Eq}(6)}$ & $2.5 \mu \mathrm{m}$ \\
\hline Springs (SiRN) & \\
\hline Length & $75 \mu \mathrm{m}$ \\
\hline Width & $10 \mu \mathrm{m}$ \\
\hline Thickness & $1 \mu \mathrm{m}$ \\
\hline Hairs (SU-8) & \\
\hline Length & $980 \mu \mathrm{m}$ \\
\hline Diameter & $50 \mu \mathrm{m}$ \\
\hline
\end{tabular}

With electrical actuation a small amplitude frequency sweep superimposed on a bias voltage was applied to the electrodes of the sensors. From the response of the sensor the resonance frequency can be derived. With the acoustic measurements actuation was done by means of a loudspeaker. Here the sample is placed in the very near field of the loudspeaker to make the loudspeaker induced particle velocity directly related to the cone velocity and the transfer independent of the driving frequency [7]. Deflection of the sensor membranes was characterized using a Polytec MSA-400 laser vibrometer. The measurement setup for the acoustic measurements is depicted in Fig. 4.

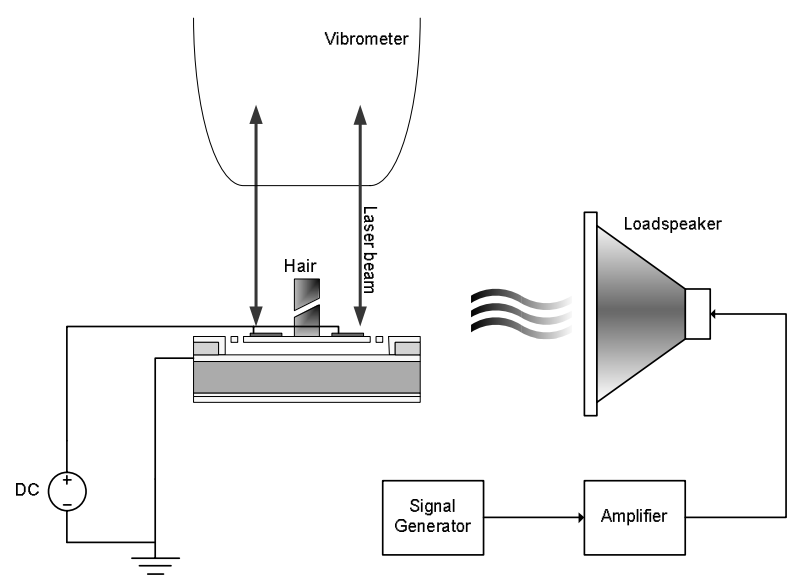

Fig. 4 Setup using loudspeaker as actuator and vibrometer for detection

\section{RESULTS}

Fig 5. shows the measured resonance frequentcies for the sensor at different applied DC-bias voltages when electrically actuated. The solid line is the model, Eq (3), fitted to the measurement data using $U_{\text {bias }}=0$, resulting in a value for $\kappa / S_{0}$ of $0.016 \mathrm{~V}^{-2}$.

\section{Frequency versus voltage}

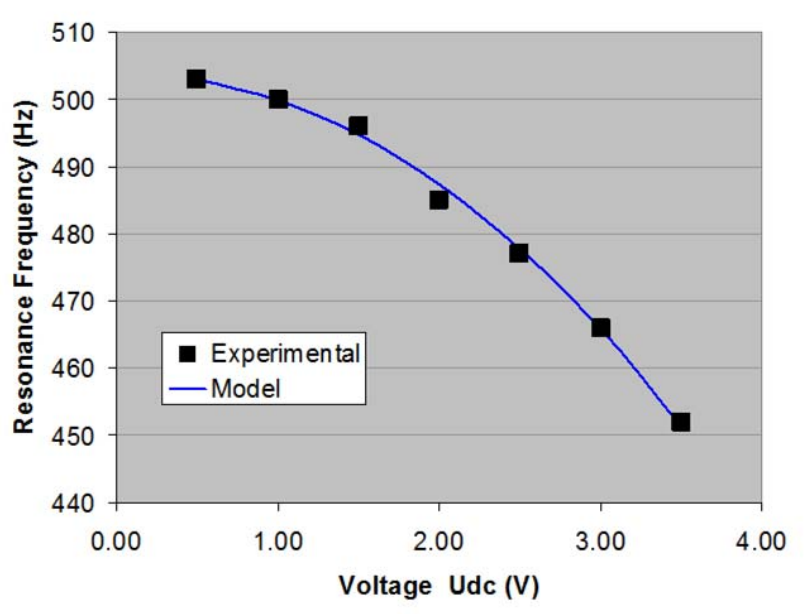

Fig. 5 Resonance frequency versus DC-bias. Solid line is the model fitted $\left(U_{\text {bias }}=0\right)$.

Fig. 6 gives the measured maximum membrane rotation normalized to the applied air flow from the loudspeaker versus the bias voltage at various actuation frequencies. 


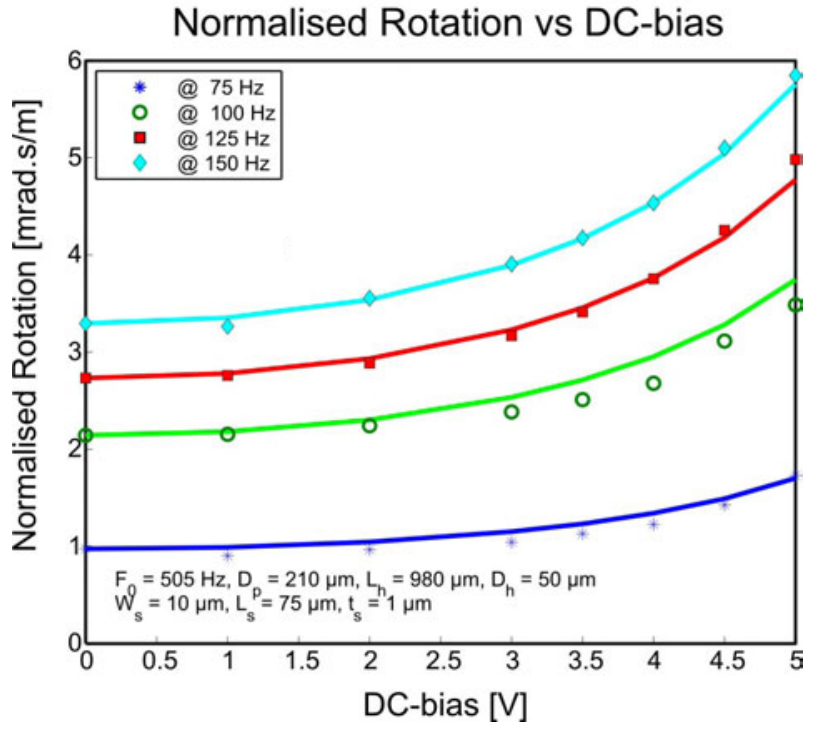

Fig. 6 Normalized rotation of the membrane due to acoustically induced flow, vs. DC-bias. The model parameters are indicated in the figure.

Fitting the measurement data to the model, Eq (2), we find a value for $\kappa / S_{0}$ of $0.0171 \mathrm{~V}^{-2}$.

\section{DISCUSSION}

In Fig. (5) and (6) we see that the trend for the resonance frequency reduction and the increased sensitivity respectively are represented very well by the model. For a comparison of the experiments and theory we calculate $\kappa$ analytically to be $104 \cdot 10^{-12} \mathrm{NmV}^{-2} . S_{0}$ can be derived from the resonance frequency and the (calculated) inertial moment of the sensor and is found to be $7.50 \cdot 10^{-9} \mathrm{Nm} / \mathrm{rad}$. We now find a value for $\kappa / S_{0}$ of $16.7 \cdot 10^{-3} \mathrm{~V}^{-2}$ which is in good agreement with the fitted values from the experimental data.

\section{CONCLUSIONS}

Adaptive behavior of the artificial hair sensors has been successfully shown. Both a reduction of the resonance frequency as well as an increase in sensitivity for air flow was measured when applying a DC-bias voltage. The theory to describe the DCbias induced adaptation was presented and shown to be in good agreement with experiments.

\section{ACKNOWLEDGEMENTS}

The authors want to thank: Meint de Boer and Erwin Berenschot for their advice on processing, Dominique Altpeter for SU-8 processing, Marcel Dijkstra for generating SEM pictures and our colleagues in the EU project CILIA for stimulating discussions and input to this work. The Customized Intelligent Life-Inspired Arrays project is funded by the Future and Emergent Technologies arm of the IST Programme.

\section{REFERENCES}

[1] Photo courtesy G. Jeronimidus, R. Seidel, K. Winwood, Biomimetics Centre, Reading University

[2] T. Shimozawa, et al. "Cricket wind receptors: thermal noise for the highest sensitivity known", in "Sensors and Sensing in Biology and Engineering", ed. Barth, Humphry and Secomb, Springer, Vienna, 2003, ISBN 3-21183771-X.

[3] See e.g. J. Levin and J. Miller, "Broadband neural encoding in the cricket cercal sensory system enhanced by stochastic resonance", Nature, vol. 380, March 1996, p. 165.

[4] Gijs J M Krijnen et al. "MEMS based hair flowsensors as model systems for acoustic perception studies", Nanotechnology, vol 17, pp. 8489, 2006

[5] T. Steinmann et al. "Air-flow sensitive hairs: boundary layers in oscillatory flows around arthropod appendages", J. Exp. Biology 209, pp 4398-4408, 2006.

[6] J.G.E Gardeniers et al.: 'LPCVD silicon-rich silicon nitride films for applications in micromechanics, studied with statistical experimental design', J. Vac. Sci. Technol. A, 1996, 14, (5), pp. 2879-2892

[7] H-E. de Bree et al. $11^{\text {th }}$ Int. Cong. on Sound and Vibration (ICSV11), St. Petersburg 2004. 